\title{
Control of a Nonlinear Tank Process using Dynamic Matrix Controller
}

\author{
M.Mirudubaashini ${ }^{1}$, Jayaprasanth $\mathrm{D}^{2}$ \\ \{mirudubaashinibarbie@gmail.com ${ }^{1}$,djp.ice@psgtech.ac.in ${ }^{2}$ \} \\ PG Scholar, Department of Instrumentation and Control Engineering, PSG College of technology, \\ Coimbatore, India ${ }^{1}$, Assistant professor (Sr. Gr.), Department of instrumentation and control \\ engineering, PSG College of technology, Coimbatore, India ${ }^{2}$
}

\begin{abstract}
The level process is widely used in many process industries for storing the liquids for mixing purpose in sugar mills, as settling tank and for water treatment plants. Most of the chemical plants are multivariable processes which exhibit nonlinear behaviour due to the existence of interactions between the input and output variables. The level control of the cylindrical tank is difficult because of its nonlinearity. Linear PID (Proportional Integral Derivative) controllers are generally used in many industrial control systemsbecause of its simple structure and robustness, but whenit comes to the control of nonlinear and multivariable processes, the controller parameters have to be continuously adjusted in order to deal with the nonlinearities, uncertainties and interaction betweenthevariables. The major objective of this work is to control the level of a nonlinear tank process. This is achieved by modelling a nonlinear controller which can adapt to the nonlinear performanceof the process. To achieve this goal, the mathematical modelling of a tank process is obtained. Dynamic Matrix Control (DMC) Controller is designed using the mathematical model to control the tank system. The performance of the controller can be analyzedthrough extensive simulation studies.
\end{abstract}

Keywords: DMC, PID.

\section{Introduction}

The control algorithm is quite complicated for the MIMO system due to variation in process dynamics that occurs due to changes that occur in the operating point and the characteristics of nonlinear dynamic coupling. Most of the real time systems are found to be nonlinear. The Tank Level System is widely used in many process industries for storing the liquids. Most of the chemical plants are multivariable processes which exhibit nonlinear behaviour due to the existence of interactions between the input and output variables. The level control of the cylindrical tank is difficult because of its nonlinearity.

The Model Predictive Control is used to compute the control signal which best corresponds to some standardthat predicts the systembehaviour.MPC is widely used for a long time in process industries. MPC has established itself in industries an important form of advanced multivariable control. The use of MPC algorithm is used from early 1970's. The first MPC algorithm designed was Model Predictive Heuristic Controlin 1976 by Richaletet al, and Cutler and Remakerin 1979 proposed Dynamic Matrix Control (DMC), the control outputs were calculated by applying the principle of receding horizon. DMC is designed for the control of level for the two conical tank interacting system and being implemented for the 
same [1]. Takagi Sugeno fuzzy expert model based soft fault diagnosis for two tank interacting system from where the mathematical model is obtained [2].

A nonlinear Hammerstein-Wiener model predictive controller based on LSSVM is built to describe the dynamic characteristic of a continuous stirred tank reactor (CSTR) from [3].The MPC algorithm is being designed for the mathematical model of the coupled tank system, which is a Multiple Input Multiple Output (MIMO) system on a flow controller valveDynamic Matrix Control (DMC) of Quadraple-Tank Process, which deals with the Model Predictive Control (MPC) known as DMC applied to a non linear multivariable laboratory for interacting tank systems [5-7].

\section{Nonlinearsystem Description}

Cylindrical tanks are broadly used in industries for storing the liquids (kerosene, petrol, water, diesel etc.), mixing purpose in sugar mills, reactors for mass chemicals separation, as settling tank and as well as for water treatment plants. While most of the chemical plants are multivariable processes which reveal nonlinear behaviour due to the continuation of interactions between input and output variables. The control of these processes is a exigent task when compared to SISO processes.

The schematic diagram of the nonlinear tank process set-up is shown in Fig.1. It consists of a cylindrical. The pneumatic control valves fine-tune the flow of water pumped from the reservoir into the tanks (Fin). Rotameters are set at the inlet the tank to measure the inflow of the tank. The tank is provide with a Differential Pressure Transmitter (DPT) to measure the pressure of the liquid column and transformed into the water level.

The discharge flow of the tank is shown in the dynamic model, the inward mass flows Finare defined as inputs, while the measurements $H(t)$ the height of fluid in tank are considered as output. The dynamic model is derived using the inward and extrovert mass flows and is described by the following differential equation (1) which is obtained from [2].

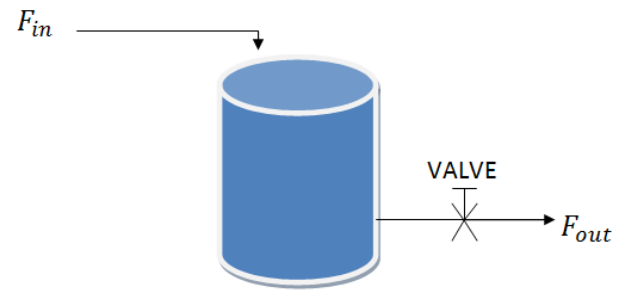

Fig 1 Nonlinear Cylindrical Tank System

\section{Mathematical Model Of Nonlinear Cylindrical Tank System}

The equation of the Nonlinear Tank process is givenby,

$$
A \frac{d h}{d t}=F_{i n}-b \sqrt{h}
$$




$$
b=s \cdot a \cdot \sqrt{2 g}
$$

Table 1 Parameters of cylindrical tank system

\begin{tabular}{|l|l|l|}
\hline S.No. & Description & Value \\
\hline $\mathbf{1}$ & $\begin{array}{l}\text { Area of the tanks } \\
\text { (A) }\end{array}$ & $0.0154 \mathrm{~m} 2$ \\
\hline $\mathbf{2}$ & $\begin{array}{l}\text { Acceleration due to } \\
\text { gravity (g) }\end{array}$ & $9.81 \mathrm{~m} / \mathrm{sec} 2$ \\
\hline $\mathbf{3}$ & $\begin{array}{l}\text { Maximum } \\
\text { permissible height of } \\
\text { water levels } \\
\text { (h max) }\end{array}$ & $0.63 \mathrm{~m}$ \\
\hline $\mathbf{4}$ & $\begin{array}{l}\text { Cross section of the } \\
\text { connecting pipes (a) }\end{array}$ & $0.005 \mathrm{~m} 2$ \\
\hline $\mathbf{5}$ & $\begin{array}{l}\text { Co-efficient of the } \\
\text { connecting pipes(s) }\end{array}$ & 0.45 \\
\hline $\mathbf{6}$ & $\begin{array}{l}\text { Nominal operating } \\
\text { condition } \\
\text { h }=0.4(\mathrm{~m})\end{array}$ & Nominal inflow rate \\
\hline
\end{tabular}

The transfer function for tank obtained from the above parameters is given as,

$$
\frac{H(s)}{F_{\text {in }}(s)}=\frac{1.2693}{1.954 s+1}
$$

\section{Dmc Controller Design}

The Model Predictive Control is the backbone of Dynamic Matrix Control. In this controller, the model which is used determines the behaviour of complex dynamical systems. The nonlinearities in the process and the complexity are due to non-coherent process degeneration. Hence, the process model predicts the behaviour of the outputs of the modelled dynamic system based on the process input changes. In this controller the transfer function needs to be specified in order. The designing is first based on the step response which is given in the form,

$$
\hat{Y}_{k}=\sum_{i-1}^{N} s_{i} \Delta u_{k-1}+s_{N} u_{k-N}
$$

The past control moves and the future control moves are seperated by the following equation,

$$
\hat{Y}_{k}=s_{1} \Delta u_{k+j-1}+s_{2} \Delta u_{k+j-2}+\cdots+s_{j} \Delta u_{k}+s_{N} \Delta u_{k-N-j}
$$




$$
+s_{j-1} \Delta u_{k-1}+s_{j+1}+s_{N-1} \Delta u_{k-N+j+2}(5)
$$

A control horizon of $\mathrm{M}$ steps and a prediction horizon of $\mathrm{P}$ steps, are written in matrixvector form, using matrix-vector notation.

$\hat{Y}_{c}=s_{f} \Delta u_{f}+s_{\text {past }} \Delta u_{\text {past }}+s_{N} u_{p}+\hat{d}$

The forced response and the free response which for forced response corresponds to the current and future control movescontributions and for free response that corresponds to the prediction of changes in output if there are no future control moves.Hence the minimized solution for this objective function is given as,

$$
\begin{aligned}
& \Delta u_{f}=\left(s_{f}^{T} s_{f}+w\right)^{-1} s_{f}^{T} E(7) \\
& \Delta u_{f}=k_{1} E(8)
\end{aligned}
$$

The equation (8) the represents the first row of the K matrix which is taken from [8].

Hence from these equations, the DMC is designed for the process model. The unforced error vector (E)is proportional to thepresent and future control move vector $\left(\Delta \mathrm{u}_{\mathrm{f}}\right)$. In example, a controller gain matrix, $\mathrm{K}$, multiplied with the unforced error vector.The first row of the $\mathrm{K}$ matrix based on the equation (8) is used as only the present control move is considered.

\section{Simulation Results}

Linearization is usually done for the system that is being non-linear to obtain the characteristic linear form of equation.

The tank process of the system is been linearized by Taylor Series for the tank equation. The Taylor series for the equation (1) of the tank process is been linearized and the transfer function is been obtained as shown in equation (3) and the Taylor series for the system to be obtained because the steady state point of operation. The equation (9) is a nonlinear equation and hence it needs to be linearized. Hence, in order to linearize the nonlinear term of the equation (1),Taylor series expansion is carried out as the following:

$$
\sqrt{h}=\sqrt{h_{s}}+\frac{1}{2 \sqrt{h_{s}}}(h)
$$

The closed loop response of the tank process is obtained using the DMC are discussed below. 


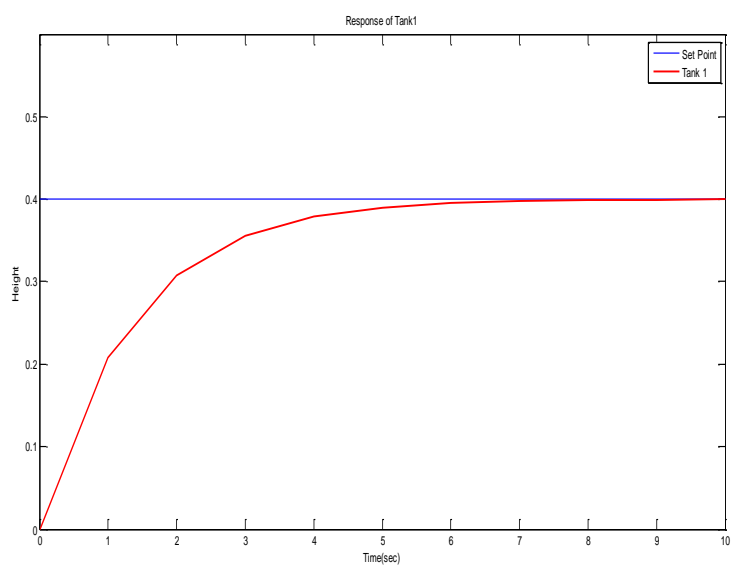

Fig.2 Closed Loop Response of Tank with $\mathrm{P}=5, \mathrm{~N}=25$

The closed-loop response at set point $\mathrm{h}=0.4 \mathrm{~m}$; prediction horizon, $\mathrm{P}=5$; control horizon, $\mathrm{M}=1$; no weighting on inputs and model length, $\mathrm{N}=25$ is shown in the Fig.2. The response is reached at a nominal speed.

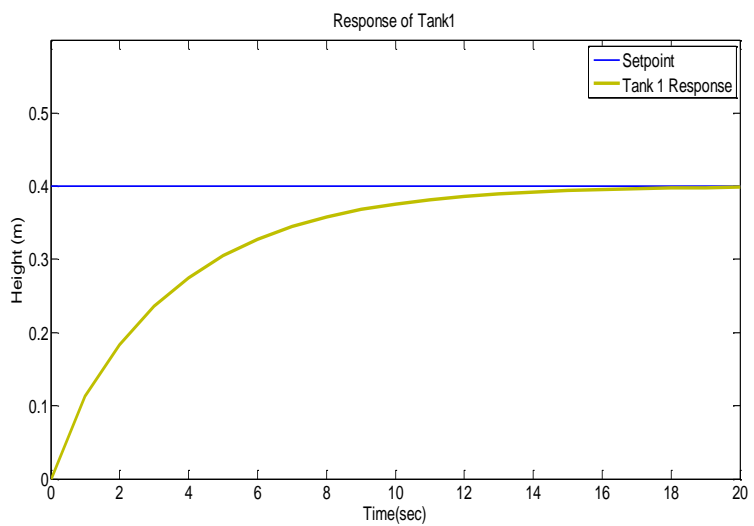

Fig 3 Closed loop response of Tank with $P=50, N=100$

The closed-loop response at set point $\mathrm{h}=0.4 \mathrm{~m}$; prediction horizon, $\mathrm{P}=50$; control horizon, $\mathrm{M}=1$; no weighting on inputs and model length, $\mathrm{N}=100$ is shown in the Fig.3. 


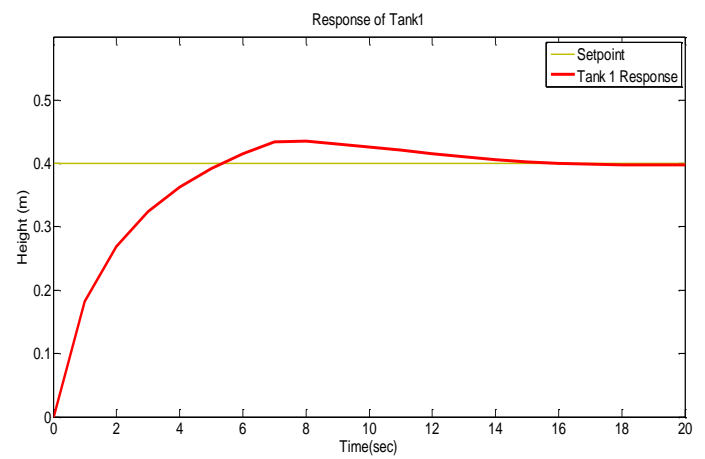

Fig.4Closed loop response of Tank with $P=5, N=6$

The closed-loop response at set point $\mathrm{h}=0.4 \mathrm{~m}$; prediction horizon, $\mathrm{P}=5$; control horizon, $\mathrm{M}=1$; no weighting on inputs and model length, $\mathrm{N}=6$ is shown in the Fig.4. The response is obtained with some deviation.

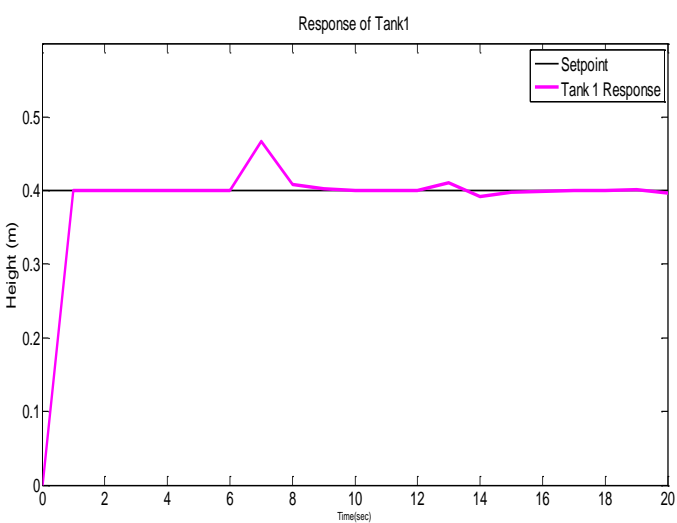

Fig.5Closed loop response of Tank with $P=1, N=6$

The closed-loop response at set point $\mathrm{h}=0.4 \mathrm{~m}$; prediction horizon, $\mathrm{P}=1$; control horizon, $\mathrm{M}=1$; no weighting oninputs and model length, $\mathrm{N}=6$ is shown in the Fig.5. The response is obtained with some overshoot after few seconds.

\section{Conclusion And Future Work}

The design of DMC was done using MATLAB and response for a first order system was obtained with different values of Model Length $(\mathrm{N})$, Control horizon $(\mathrm{M})$ and Prediction horizon $(\mathrm{P})$. The $\mathrm{P}$ should be lower than $\mathrm{N}$ otherwise the response reaches the steady state slowly. The same controller design was carried for the Tank process and the different values of $\mathrm{P}$ and $\mathrm{N}$ was given for the unit $\mathrm{M}$ value but with no weighting. It was observed that the $\mathrm{P}$ having higher value gives a slower settling time and the from the model length, it is observed that for lower values of Ndoes not capture the process dynamics completelyand thereby resulting in poor performance. 
In future, the two-tank nonlinear interacting system can be carried out as a future work using DMC by selecting appropriate prediction horizon, control horizon and the model length. Then this work can be extended to the real time process.

\section{References}

[1] V. R. Ravi, T. Thyagarajan and G. Uma Maheshwaran, "Dynamic Matrix Control of Two Conical Tank Interacting Level System", International Conference on Modelling Optimization and Computing (ICMOC- 2012)., Procedia Engineering Elsevier., vol. 38, pp. 2601-2610, 2012.

[2] Pandiyan Manikandan, Mani Geetha, "Takagi sugeno fuzzy expert model based soft fault diagnosis for two tank interacting system ", International journals on Achieves of Control science., vol. 24, no. 3, pp. 271-287, 2014.

[3] Man Hong and Shao Cheng, "Hammerstein-Weiner Model Predictive Control of Continuous Stired Tank Reactor", Electronics and Signal Processing, Springer, vol. 97, pp. 235-242, 2011.

[4] Liankui Dai and Karl Johan Astrom, "Dynamic Matrix Control of a Quadruple-Tank Process", 14 World Conference of International Federation of Automatic Control, ,Procedia Engineering Elsevier, vol. 32, no. 2, pp. 6902-6907, 1999.

[5] L. ThillaiRani, N. Deepa and S. Arulselvi, "Modelling and Intelligent Control of Two-Tank Interacting Level Process", International Journal of Recent Technology and Engineering. vol. 3, no.1, pp. 277-3878, March 2014.

[6] Nayanmani Deka and Dr. Lini Mathe, "PID Controller for two tank liquid process using LabVIEW", International Research Journal for Science and Computing. vol. 7, no. 5, 2017.

[7] Damrudhar and Dr. D.K. Tanti, "Comparitive Performance Analysis for Two Tank Liquid Level Control System with Various Controllers using Matlab", vol. 7, no. 2, July, 2016.

[8] B. Wayne Bequette, Process Control: Modeling, Design and Simulation, Pearson Education Inc, USA, 2003. 